part of the sensory system might lead to an affection generalised over the whole of that system; although it is impossible to assert without evidence from morbid anatomy, that is not at present forthcoming, how that generalisation is effected. But, in their opinion, clinical evidence establishes the fact.

The interval between the injury and the first symptoms of tabes varied from a fow weeks to as much as eighteen years; and it is worthy of note that, in five out of the fourteen cases quoted, nervous heredity, alcoholism or syphilis were stated to be present. Former writers have noticed an occasional connection between injuries and locomotor ataxy; and Erb (quoting Schulze) cites cases in which it has followed fracture of the thigh or a fall on the abdomen. Petit considers that wounds at a distance " hasten its development," and others have counted injuries in a general way as among the causes of tabes. The present writers carry this view much further. Given a personal or hereditary predisposition to nervous affection, they hold that, besides fractures, many slight injuries, such as sprains or wounds of the feet, or even operations in dentistry or for cataract, may be sufficient determining causes. Again, the first symptom of the tabes arises always, in their view, in the injured region. After injury to the left foot, the symptoms commenced after five months with lightning pains in the same limb; after contusion of the abdomen, with gastric crises, two months from the date of injury. This circumstance will often afford a guide in the history of each case to the original exciting cause. Finally it is allowed that diatheses-alcoholism, syphilis or rheumatism, may exert in some of these cases a predisposing influence.

Fortescue Fox, M.D.

Raymond on Sweating of the Face (Arch. de Neurol., Jan. 1888).-The first case recorded is that of a man, aged 31, who noticed in 1880 that sweating of the right side of the face.always appeared two or three minutes after he had taken food, and ceased when he left off eating. The sweating extended on to the' neck and as far as the shoulder. Hot food induced it most rapidly; movements and the idea of eating had no effect; all other-symptoms were absent, except that the right pupil was dilated and not affected by light, and below the right wrist there was difficulty in distinguishing between hot and cold objects. Injections of two milligrammes of pilocarpin produced a local sweating of the face on the right. Neither galvanism, nor atropine, nor ergotine, nor tannin produced any effect on the patient's malady.

The author divides the cases of local sweating into the following 


\section{ABSTRACTS OF BRITISH AND FOREIGN JOURNALS.}

groups. First:-Those in which there is an alteration in the cerebrospinal nervous system. Second:-Those in which the cervical sympathetic, or the first thoracic ganglion is affected. As a rule, in these cases, the pupil is contracted, but one is cited in which it was dilated. In this case there was chronic inflammation of the superior cervical ganglion on the same side. The author's second case is placed in this second group ; it is that of a man, aged 39 , who had phthisis ; he also had unilateral right-sided sweating, especially of the forehead. There were no vaso-motor or pupillary symptoms. He died suddenly, three days after an epileptiform attack. The tubercular pleurisy had bound down and caused chronic inflammatory change in the right inferior cervical ganglion. Third:Those in which the nerves of the face are affected. Fourth :-Those in which the sweating is reflex. These cases usually occur in nervous hysterical people. Many examples of this group are given, such as that of a young man in whom facial sweating was induced by smelling vinegar.

Thus there are two classes of cases characterised by increased sweating; those in which there are and those in which there are not vaso-motor troubles. The lesion in the former is in the sympathetic of the neck ; the fact that the pupil is sometimes dilated and sometimes contracted depends upon the fact, that the pupillary and vaso-motor nerves are probably distinct, and one set may be stimulated whilst the other is paralysed. The author then gives an account of the various chronic inflammatory changes that have been found in the superior cervical ganglion, and concludes that these irritate the sweat-secreting nerves. Sometimes these changes exist without there being any alteration of sweating. Lastly, he points out, that the pupillary changes are permanent whilst the sweat ones are transitory; that the increased secretion of sweat may extend beyond the face, so that treatment has very little effect.

HALE WhITE, M.D. 